\title{
Standardized Index of Shape (DCE-MRI) and Standardized Uptake Value (PET/CT): Two quantitative approaches to discriminate chemo-radiotherapy locally advanced rectal cancer responders under a functional profile
}

\author{
Antonella Petrillo ${ }^{1}$, Roberta Fusco ${ }^{1}$, Mario Petrillo ${ }^{1}$, Vincenza Granata ${ }^{1}$, Paolo \\ Delrio $^{2}$, Francesco Bianco ${ }^{2}$, Biagio Pecori ${ }^{3}$, Gerardo Botti ${ }^{4}$, Fabiana Tatangelo ${ }^{5}$, \\ Corradina Caracò, Luigi Aloj ${ }^{6}$, Antonio Avallone7, Secondo Lastoria6 \\ ${ }^{1}$ Radiology Unit, Department of Diagnostic Imaging, Radiant and Metabolic Therapy, "Istituto Nazionale Tumori Fondazione \\ Giovanni Pascale - IRCCS", 80131, Naples, Italy \\ ${ }^{2}$ Gastrointestinal Surgical Oncology Unit, Department of Abdominal Oncology, "Istituto Nazionale Tumori Fondazione Giovanni \\ Pascale - IRCCS", 80131, Naples, Italy \\ ${ }^{3}$ Radiotherapy Unit, Department of Diagnostic Imaging, Radiant and Metabolic Therapy, "Istituto Nazionale Tumori Fondazione \\ Giovanni Pascale - IRCCS", 80131, Naples, Italy \\ ${ }^{4}$ Scientific Director, "Istituto Nazionale Tumori Fondazione Giovanni Pascale - IRCCS", 80131, Naples, Italy \\ ${ }^{5}$ Diagnostic Pathology Unit, Department of Diagnostic and Laboratory Pathology "Istituto Nazionale Tumori Fondazione \\ Giovanni Pascale - IRCCS", 80131, Naples, Italy \\ ${ }^{6}$ Nuclear Medicine Unit, Department of Diagnostic Imaging, Radiant and Metabolic Therapy, "Istituto Nazionale Tumori \\ Fondazione Giovanni Pascale - IRCCS", 80131, Naples, Italy \\ ${ }^{7}$ Gastrointestinal Medical Oncology Unit, Department of Abdominal Oncology, "Istituto Nazionale Tumori Fondazione Giovanni \\ Pascale - IRCCS", 80131, Naples, Italy \\ Correspondence to: Antonella Petrillo, email: a.petrillo@istitutotumori.na.it \\ Keywords: rectal cancer, neoadjuvant chemo-radiotherapy, DCE-MRI and FDG-PET/CT, treatment response assessment \\ Received: August 26, $2016 \quad$ Accepted: November 21, $2016 \quad$ Published: December 22, 2016
}

\section{ABSTRACT}

Purpose: To investigate dynamic contrast enhanced-MRI (DCE-MRI) in the preoperative chemo-radiotherapy (CRT) assessment for locally advanced rectal cancer (LARC) compared to ${ }^{18} \mathrm{~F}$-fluorodeoxyglucose positron emission tomography/computed tomography ( ${ }^{18}$ F-FDG PET/CT).

Methods: 75 consecutive patients with LARC were enrolled in a prospective study. DCE-MRI analysis was performed measuring SIS: linear combination of percentage change $(\Delta)$ of maximum signal difference (MSD) and wash-out slope (WOS). ${ }^{18}$ F-FDG PET/CT analysis was performed using SUV maximum (SUV $\mathrm{max}_{\text {m }}$ ). Tumor regression grade (TRG) were estimated after surgery. Non-parametric tests, receiver operating characteristic were evaluated.

Results: 55 patients (TRG1-2) were classified as responders while 20 subjects as non responders. $\Delta$ SIS reached sensitivity of $93 \%$, specificity of $80 \%$ and accuracy of $89 \%$ (cut-off $6 \%$ ) to differentiate responders by non responders, sensitivity of $93 \%$, specificity of $69 \%$ and accuracy of $79 \%$ (cut-off $30 \%$ ) to identify pathological complete response ( $P C R$ ). Therapy assessment via $\Delta \mathbf{S U V}_{\text {max }}$ reached sensitivity of $67 \%$, specificity of $75 \%$ and accuracy of $70 \%$ (cut-off $60 \%$ ) to differentiate responders by non responders and sensitivity of $80 \%$, specificity of $31 \%$ and accuracy of $51 \%$ (cut-off $44 \%$ ) to identify PCR.

Conclusions: CRT response assessment by DCE-MRI analysis shows a higher predictive ability than ${ }^{18} \mathrm{~F}-\mathrm{FDG}$ PET/CT in LARC patients allowing to better discriminate significant and PCR. 


\section{INTRODUCTION}

Approximately forty thousand new cases of rectal cancer are accounting in the USA in 2015 [1]. Despite the introduction of the screening programs, several patients are diagnosed in a locally advanced stage. Preoperative radiochemotherapy (pCRT) associated with total mesorectal excision (TME) is the standard care procedure for locally advanced rectal cancer (LARC) [2,3]. TME is linked to morbidity and complications, therefore in clinical practise there is an increase of conservative treatment strategies application for patients with substantial tumor regression after pCRT and "wait and see" policy for patients with complete pathological regression. The advantage of this strategy is the reduction of morbidity and the possibility to provide a "true" organ-sparing approach. In this scenario is necessary to individuate the selection criteria for these strategies that accurately can assess neoadjuvant treatment response. Morphological MRI (mMRI) is the best tool for local LARC staging, permitting a correct assessment of the disease extent, of the mesorectal fascia and lymph node involvement $[4,5]$. On the other hand, there are some limits to detect changes after pCRT by means of mMRI [4]. A positive tumor response may not correspond to a significant tumor size reduction. Moreover, it is difficult to discriminate between post treatment fibrosis and residual viable tumor using morphological approach. To overcome this limitation, functional approaches that aim to assess tissue "viability" through different imaging modalities such as Position Emission Tomography, Dynamic Contrast Enhanced-Magnetic Resonance Imaging (DCE-MRI), Diffusion Weighted Magnetic Resonance Imaging (DWI) are being actively investigated. One widely used approach is Positron Emission Tomography coupled with Computed Tomography (PET/CT) that in rectal cancer management is capable to early predict treatment response [6-10]. However, among data reported in literature [7-8, 10], late PET scans, performed before surgery, showed lower accuracy in pathologic response assessment.

Some authors described the value of mMRI and additional ${ }^{18} \mathrm{~F}$-fluorodeoxyglucose positron emission tomography/computed tomography $\left({ }^{18} \mathrm{~F}-\mathrm{FDG} \mathrm{PET} / \mathrm{CT}\right)$ for pCRT tumor response evaluation in patients with LARC [7, 8]. In Huh et al. [7] sensitivity, specificity and diagnostic accuracy of mMRI to predict pathologic complete response were $38.5 \%, 58.1 \%$ and $55.2 \%$, respectively. Using a response index (percentage change of Standardized Uptake Value maximum, $\Delta$ SUVmax) of $63.6 \%$, it was possible to detect the complete response response with a sensitivity of $73.1 \%$, a specificity of $64.5 \%$ and an accuracy of $65.7 \%$. Aiba et al. [8] have shown no benefit adding ${ }^{18} \mathrm{~F}-\mathrm{FDG}$ PET/CT to $\mathrm{mMRI}$ in assessment of pCRT responders based on changes in area under receiver operating characteristic curve. To the best of our knowledge, there are no available studies in the literature on an enough number of patients that directly compare functional parameters obtained by ${ }^{18} \mathrm{~F}-\mathrm{FDG}$ PET and DCE-MRI in the pre-surgical evaluation of CRT in LARC. Using these imaging methods with the same timing allows exploring potential existing relationships between two different functional tissue proprieties: tumor vascularity investigated by tissue perfusion and tissue glucose metabolism [11-25].

In a previous study, we investigated a semiquantitative analysis with DCE-MRI [14-20], finding the best combination, denominated Standardized Index of Shape (SIS), that identifies the linear classifier of the percentage differences $\Delta$ of Maximum Signal Difference (MSD) and of Wash-Out Slope (WOS) [7], with a sensitivity and specificity of $93.5 \%$ and $82.1 \%$ in discrimination of responder by non responder patients after pCRT [13].

The objective of this study was to validate the potential of SIS analysis in LARC to identify significant and pathological complete response after neoadjuvant preoperative CRT, in comparison with ${ }^{18} \mathrm{~F}$-FDG PET.

\section{RESULTS}

For both examinations, PET/CT and DCE-MRI, the temporal range between baseline and preoperative scan were 90 days $( \pm 15)$. The median interval between the end of CRT and TME was 9 weeks for both regimen (range, 7-14). All patients in our series had a radical resection with an undamaged and complete mesorectum. Patient characteristics and clinical staging including Gunderson Risk [25], distance by anal verge, circumferential resection margin were reported in Table 1.27 patients were classified as pT0 (pathological T), 7 as pT1, 31 as pT2, 9 as pT3, and 1 as pT4. There were 30 patients with a tumour regression grade (TRG) equal to 1,25 with a $\mathrm{TRG}=2,10$ with a $\mathrm{TRG}=3,10$ with a $\mathrm{TRG}=4$, and none with a TRG $=5$. Then, based on TRC classification $55(73.3 \%)$ patients were classified as responders $(\mathrm{TRG}=$ $1-2)$ and $20(26.7 \%)$ as non-responders $(\mathrm{TRG}=3-4)$. No significant differences between pathological responders and non-responders could be found regarding patients characteristics (Table 1).

Median values for $\Delta \mathrm{SIS}$ and $\Delta \mathrm{SUV}_{\text {max }}$ for responder and non-responder patients according to TRG (TRG 1-2 vs TRG 3-4) and pathological T (pT 0-2 vs pT3-4) are reported in Table 2. Mann-Whitney test showed statistically significant differences for $\Delta \mathrm{SIS}$ and $\Delta \mathrm{SUV}_{\max }$ median values between responders and non-responders patient based on TRG. Statistically significant differences based on pT were only found for $\Delta$ SIS values (Table 2).

Figure 1a shows ROC analysis for $\triangle \mathrm{SIS}$ and $\Delta \mathrm{SUV}_{\max }$ in discriminating responders from non responders. The optimal cut-off for $\Delta$ SIS was a reduction of $6.0 \%$ yielding $92.7 \%$ of sensitivity and $80.0 \%$ of specificity to identify responder patients. Instead, the optimal cut-off of $59.7 \%$ for $\Delta \mathrm{SUV}_{\max }$ showed lower 
Table 1: Patient Characteristics and Clinical Staging

\begin{tabular}{lcccc}
\hline Characteristics & All patients $\mathbf{n}=\mathbf{7 5}$ (\%) & TRG 1-2 $\mathbf{n}=\mathbf{5 5}$ & TRG 3-4 $\mathbf{n}=\mathbf{2 0}$ & $\mathbf{p}^{*}$ \\
\hline Gender & & & & $>0.05$ \\
Male/Female & $50(67) / 25(33)$ & $37 / 18$ & $13 / 7$ & \\
Median age (range) & $62(44-77)$ & $62(44-77)$ & $63(44-76)$ & \\
Gunderson Risk (25) & & & & $>0.05$ \\
$\quad$ Intermediate: T3N0 & $4(5.3)$ & 3 & 9 & \\
Moderately high: T3N1, T4N0 & $26(34.7)$ & 17 & 12 & $>0.05$ \\
High: T3N2, T4N1-2 & $45(60.0)$ & 33 & & \\
Distance from the anal verge & & & 9 & $>0.05$ \\
$\leq 5$ cm & $37(49)$ & 28 & 11 & \\
$>5$ cm & $38(51)$ & 27 & 2 & \\
Circumferential resection margin & & & 2 & \\
$>2$ mm & $32(43)$ & 26 & 2 & \\
$>1$ and $\leq 2$ mm & $9(12)$ & 7 & 20 & \\
$\leq 1$ mm & $30(40)$ & 2 & & \\
Not mesurable & $4(5)$ & & & \\
\hline
\end{tabular}

*Chi-square test or Mann- Whitney U Test

Table 2: $\Delta$ SIS and $\Delta S U V_{\text {max }}$ median values for patients subgroups depending on TRG and pT score

\begin{tabular}{|c|c|c|c|c|}
\hline & All patients $n=75$ & TRG 1-2 n= 55 & TRG 3-4 n=20 & $\mathbf{p}^{*}$ \\
\hline$\Delta S U V_{\max }$ median (standard deviation) & $62 \%(36)$ & $73 \%(37)$ & $48 \%(30)$ & $<0.001$ \\
\hline \multirow[t]{2}{*}{$\Delta$ SIS median (standard deviation) } & $36 \%(65)$ & $57 \%(49)$ & $-18 \%(73)$ & $<0.001$ \\
\hline & & pT 0-2 n= 65 & pT 3-4 n=10 & $\mathbf{p}^{*}$ \\
\hline$\Delta S U V_{\max }$ median (standard deviation) & & $66(36)$ & $58(34)$ & $>0.05$ \\
\hline$\Delta$ SIS median (standard deviation) & & $42(59)$ & $-20(80)$ & $<0.001$ \\
\hline
\end{tabular}

* Mann- Whitney U Test

accuracy in identifying responder patients than $\triangle \mathrm{SIS}$, with a sensitivity of $67.3 \%$ and a specificity of $75.0 \% .55$ patients were classified as responders by $\Delta \mathrm{SIS}$, including 51 true positives, while 41 patients were classified as responders by $\triangle \mathrm{SUV}_{\max }$, including 37 true positives. The combination of $\Delta$ SIS and $\Delta \mathrm{SUV}_{\max }$ did not increase predictive ability, classifying 43 patients as responders, of whom only 36 were true pathological responders. Figure $1 \mathrm{~b}$ shows ROC analysis for $\triangle \mathrm{SIS}$ and $\triangle \mathrm{SUV}_{\text {max }}$ in discrimination pathological complete response (TRG1) by incomplete response (TRG 2-4). The optimal cut-off for $\Delta$ SIS was a reduction of $30.3 \%$ (93.3\% of sensitivity and $68.9 \%$ of specificity) while the optimal cut-off of $43.9 \%$ for $\triangle \mathrm{SUV}_{\text {max }}$ showed lower accuracy (sensitivity of $80.0 \%$ and specificity of $31.1 \%$ ). Statistically significant differences between $\triangle \mathrm{SIS}$ and $\Delta \mathrm{SUV}_{\max }$, in terms of both sensitivity and specificity, were assessed using the
McNemar test ( $\mathrm{p}$ value $<0.05$ ), for both analysis. The presurgical PET/CT analysis demonstrated a low level of correlation between median $\triangle \mathrm{SUV}_{\max }$ value with $\mathrm{pT}$ and TRG findings (Spearman's rank correlation coefficient $=-0.2$ and -0.3 , respectively), while a good level of correlation was observed between median $\Delta$ SIS value and $\mathrm{pT}$ and between median $\triangle$ SIS value and TRG (Spearman's rank correlation coefficient $=-0.6$ and -0.7 , respectively). Table 3 shows the performance of $\triangle$ SIS and $\triangle \mathrm{SUV}$ analysis to identify responder from non-responder patients and complete by incomplete pathological response. Figures 2 and 3 show DCE-MRI and ${ }^{18} \mathrm{~F}-\mathrm{FDG}$ PET/CT images respectively, for a responder patient $(\mathrm{TRG}=1)$. Figures 4 and 5 respectively show DCE-MRI and ${ }^{18} \mathrm{~F}-\mathrm{FDG}$ $\mathrm{PET} / \mathrm{CT}$ images, for another responder patient $(\mathrm{TRG}=2)$ recognized only to $\Delta$ SIS analysis. 


\section{DISCUSSION}

The aim of the study was validate the potential of DCE-MRI (by means of $\Delta$ SIS value) in comparison to $\mathrm{PET} / \mathrm{CT}$ (by means of $\triangle \mathrm{SUVmax}$ ) to evaluate preoperative neoadjuvant CRT response in LARC patients. There is a growing need to optimize the multidisciplinary management of patients with LARC, considering on the one hand that tumour response and patient benefit from CRT may considerably vary and on the other that preoperative treatment and TME are not completely free from serious early and late morbidity. In this scenario, the identification of patients with TRG $1-2$, usually associated with a low prevalence of nodal involvement and a better outcome [26], would allow candidates to be selected for conservative mini-invasive strategies or for a "wait-andsee" policy [27-29].

Some authors reported the value of DCE-MRI based on semi-quantitative parameters such as initial slope, initial peak, late slope, and area under time intensity curve [30] or kinetic features (Ktrans, kep, ve) [31] in the evaluation of pathological complete response to pCRT in LARC. Martens et al. [30] concluded that "late slope" derived from DCE-MRI analysis using a semiquantitative approach could predict before the beginning of pCRT which tumors are likely going to respond. Tong et al. [31] concluded that DCE-MRI could differentiate between pathological complete and incomplete pCRT response using a Ktrans threshold value of 0.66 reaching the $100 \%$ of sensitivity. Furthermore, some studies have shown how PET evaluation can predict pathologic tumor response and outcome after preoperative CRT in
LARC patients, suggesting its great potential in assisting physicians on individualized management decisions in this disease $[7-8,10]$. Several authors studied the benefit of apparent diffusion coefficient (ADC) of DWI and SUV of PET/CT in the assessment of pCRT response in LARC [32-34] showing that their combination allows to increase the sensitivity of the correct detection of response than either approach alone. However, a systematic review [34] reported a low positive predictive value (PPV) to predict pathological complete response (PPV of $54 \%$ and $39 \%$ for DWI and PET/CT, respectively). Baseline CRT imaging is not capable to forecast pathological complete response with overall accuracies of $68-72 \%$ for DWI and $44 \%$ for PET/CT. Qualitative DWI evaluation after CRT (5-10 weeks after the end) may outperform apparent diffusion coefficient reaching an overall accuracy of $87 \%$ versus $74-78 \%$. The major strength of DWI and PET/CT is the capability to identify the non-responder patients who are not candidates for organ preservation. However, both DWI and PET/CT are not accurate enough to safely identify patients candidates for conservative mini-invasive treatments of for "wait and watch" policy allowing organsparing.

Our results show that $\Delta \mathrm{SUV}_{\max }$, between basal and pre-surgery SUV values, showed a significant correlation to TRG (AUC 0.71) with a sensitivity of $67.3 \%$, a specificity of $75.0 \%$ and an accuracy of $69.7 \%$, considering the optimal cut-off value of $59.7 \%$ provided by ROC analysis while a lower accuracy is shown to identify pathological complete response (sensitivity of $80.0 \%$ and specificity of $31.1 \%$ ). Moreover, our results showed that $\Delta \mathrm{SUV}_{\max }$ median values were statistically different at
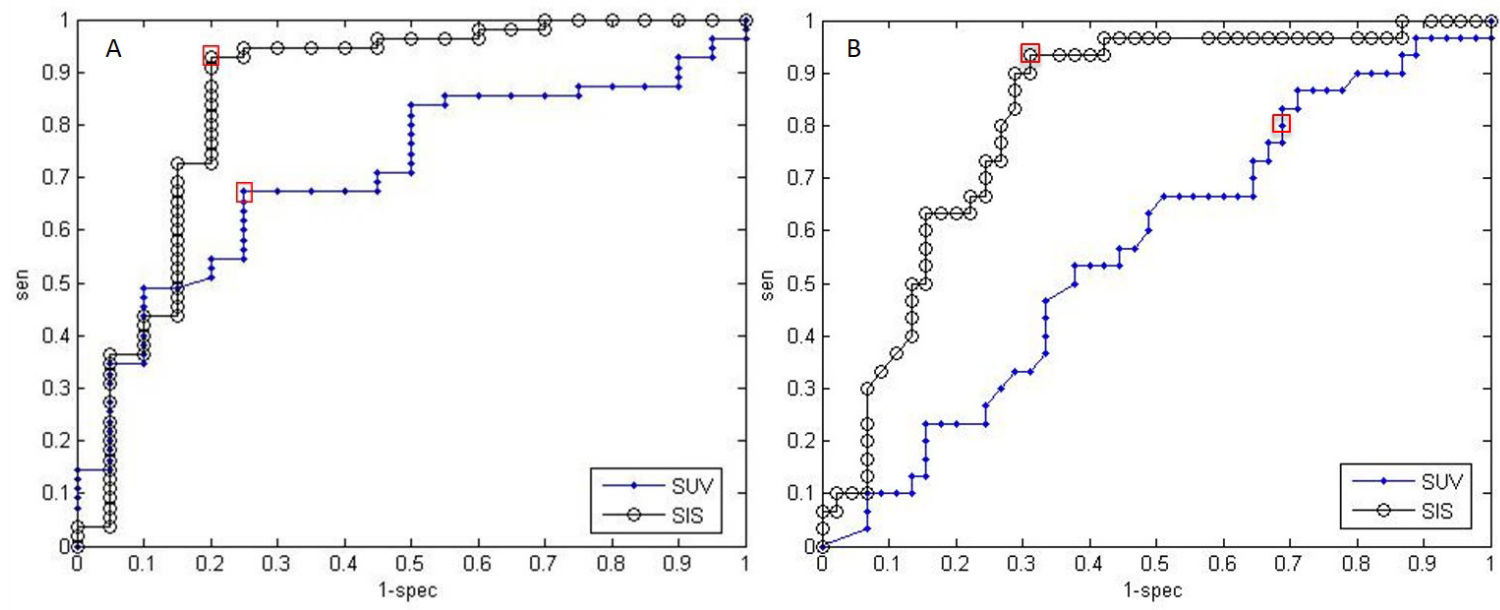

Figure 1: A. ROC analysis for $\triangle \mathrm{SIS}$ and $\Delta \mathrm{SUV}_{\max }$ in discriminating responders from non responders. Cut-off value in $\Delta \mathrm{SIS}$ (black line, $\mathrm{AUC}=0.86$ ) changed of $6.0 \%$ yields $92.7 \%$ of sensitivity and $80.0 \%$ of specificity. Cut-off value in $\Delta \mathrm{SUV}_{\max }$ (blue line, AUC $\left.=0.71\right)$ of $59.7 \%$ gave a sensitivity of $67.3 \%$ and a specificity of $75.0 \%$. B. ROC analysis for $\Delta$ SIS and $\Delta \mathrm{SUV}_{\max }$ to identify pathological complete response. Cut-off value in $\triangle \mathrm{SIS}$ (black line, $\mathrm{AUC}=0.82$ ) changed of $30.3 \%$ yields $93.3 \%$ of sensitivity and $68.9 \%$ of specificity. Cut-off value in $\triangle \mathrm{SUV}_{\max }$ (blue line, $\mathrm{AUC}=0.57$ ) of $43.9 \%$ gave a sensitivity of $80.0 \%$ and a specificity of $31.1 \%$. The two red rectangles highlight the sensitivities and specificities corresponding to the optimal thresholds. 
Table 3: Diagnostic Performance of $\Delta S I S$ and $\Delta S U V_{\text {max }}$

\begin{tabular}{|c|c|c|c|c|c|c|c|}
\hline & Cut-off \% & Sensitivity \% & Specificity \% & PPV \% & NPV \% & $\mathrm{ACC} \%$ & AUC \\
\hline \multicolumn{8}{|c|}{ To differentiate responders (TRG1-2) by non responders (TRG 3-5) } \\
\hline$\triangle S U V_{\max }$ & 59.7 & 67.3 & 75.0 & 88.1 & 45.5 & 69.7 & 0.71 \\
\hline$\triangle S I S$ & 6.0 & 92.7 & 80.0 & 92.7 & 80.0 & 89.3 & 0.86 \\
\hline \multicolumn{8}{|c|}{ To differentiate complete pathological response (TRG) by incomplete response (TRG 2-5) } \\
\hline$\triangle S U V_{\max }$ & 43.9 & 80.0 & 31.1 & 43.6 & 70.0 & 50.7 & 0.57 \\
\hline$\triangle S I S$ & 30.3 & 93.3 & 68.9 & 66.7 & 93.9 & 78.7 & 0.82 \\
\hline
\end{tabular}
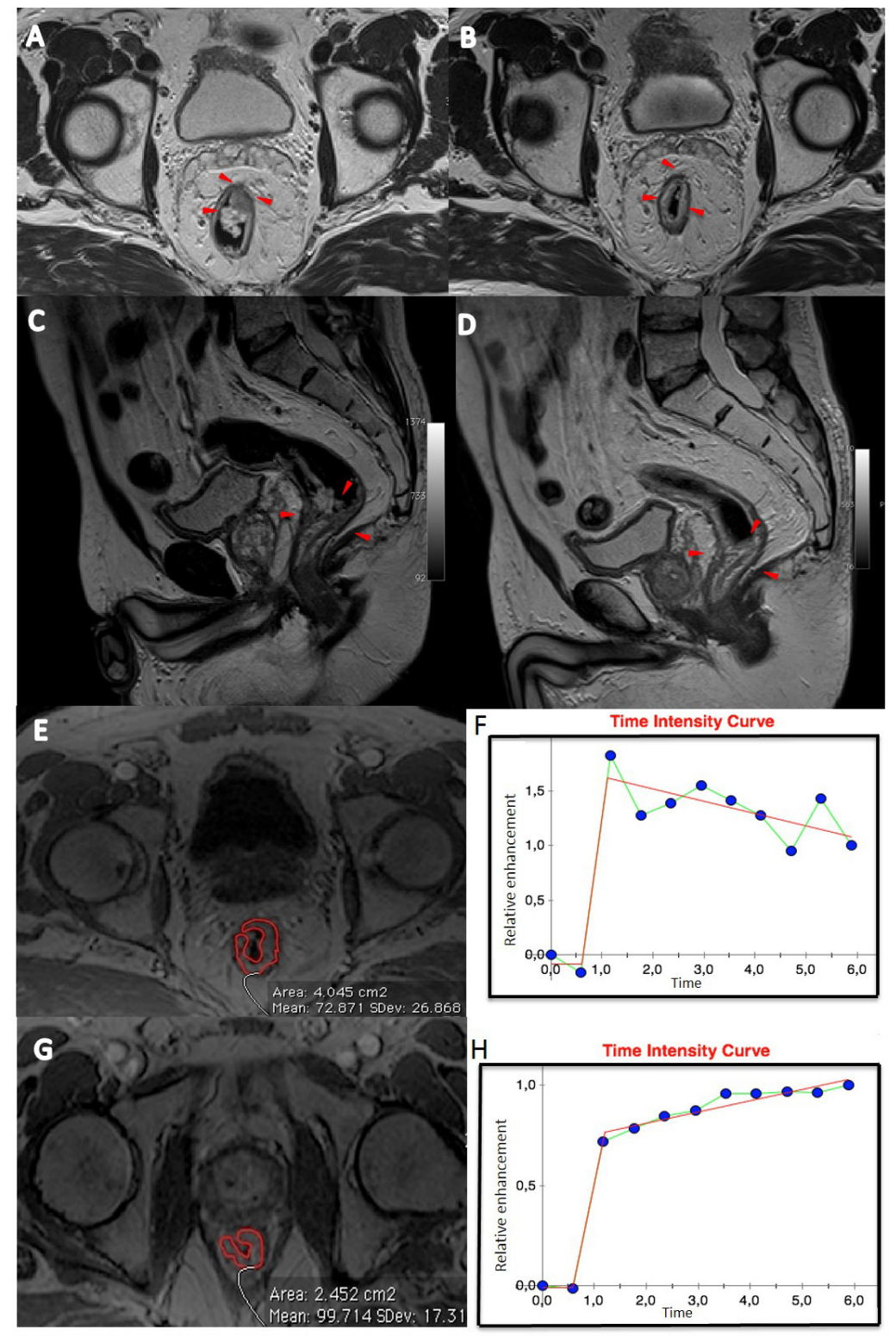

Figure 2: Patient n. 64: T2-weighted images in axial $\mathbf{A}$ and sagittal $\mathbf{C}$ plane before $\mathbf{B}$ and after treatment $\mathbf{D}$ The morphologic images (A and C) before CRT, showed heterogeneous irregular thickening along the rectal wall spreading into the perirectal fat (A, arrowheads). After CRT, a hypointense area relating to rectal wall thickening is still visible (B and D, arrowheads). Median Time intensity curve of volume of interest ( $\mathbf{E}$ and $\mathbf{G})$, segmented by expert radiologist, before treatment is shown in $\mathbf{F}$ and after treatment in $\mathbf{H}$ These curves showed different contrast enhancement, with a $\Delta$ SIS of $31.93 \%$ classifying the patient as responder. 

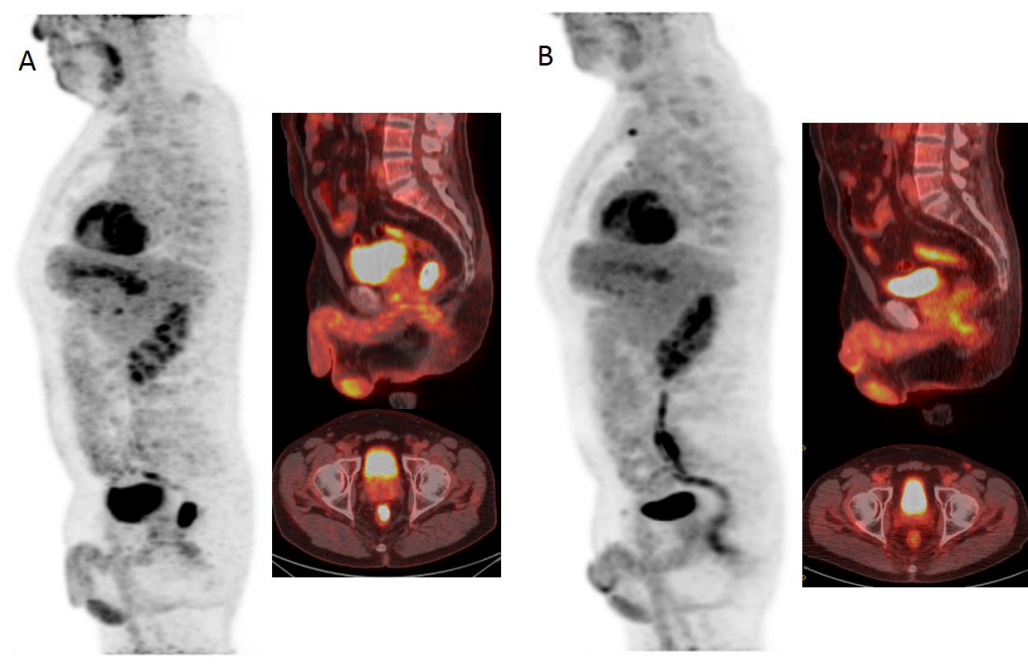

Figure 3: ${ }^{18} \mathrm{~F}-\mathrm{FDG}$ PET/CT images before $\mathbf{A}$ and after $\mathbf{B}$ treatment showed a reduction of FDG uptake with a $\Delta \mathrm{SUV}$ max 0 $96.67 \%$ classifying the patient as responder.

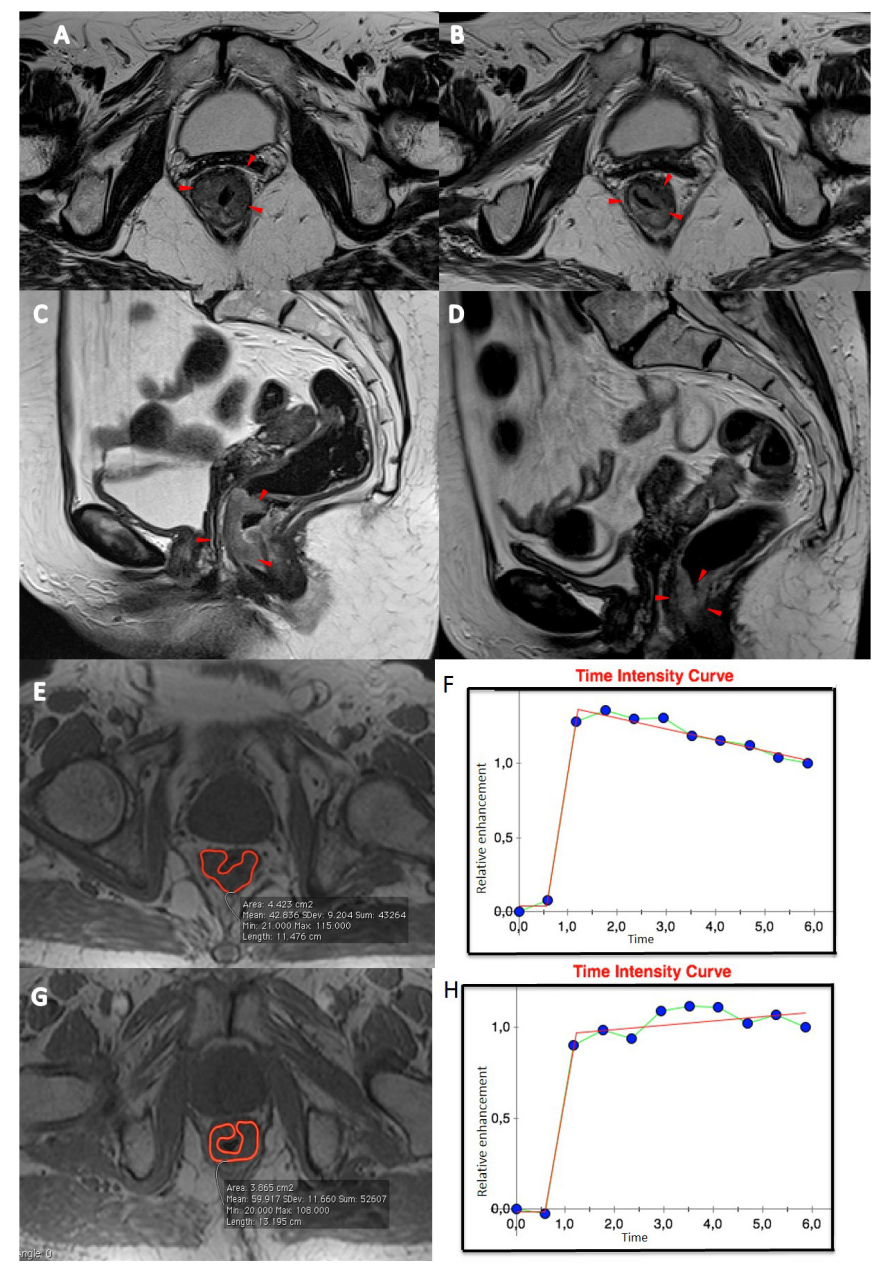

Figure 4: Patient n. 68: T2-weighted images in axial $\mathbf{A}$ and sagittal $\mathbf{C}$ plan $\mathbf{B}$ before the treatment and after treatment $\mathbf{D}$ The morphologic images (A and C) before CRT, showed heterogeneous irregular thickening along the rectal wall spreading into the perirectal fat (A, and $\mathrm{C}$ arrowheads). After CRT, a hypointense area with thickening of the rectal wall and straining into perirectal fat (B and D, arrowheads) is observed. Median Time intensity curve of the volume of interest $\mathbf{E}$ and $\mathbf{G}$, before $\mathbf{F}$ and after treatment $\mathbf{H}$ are shown. These curves showed different contrast enhancement, with a $\Delta$ SIS value of $39.54 \%$ classifying the patient as responder. 
Mann-Whitney $U$ test for responder and not responder patients based on TRG. These findings with ${ }^{18} \mathrm{~F}-\mathrm{FDG}$ PET/ CT, using Standardized Uptake Value, are in agreement with previous results [10, 35-38]. Avallone et al. [10] reported that early changes of SUVmax were predictive of pathological response with an optimal threshold value of $-42.0 \%$ and an accuracy of $93.0 \%$. In this study, the authors also observed that the findings obtained from late PET scans, performed before surgery, showed lower accuracy in predicting pathologic response. Leccisotti et al. [35] evaluated metabolic modifications in the tumour during and after pCRT in 124 patients affected by LARC. A reduction of $61.2 \%$ of SUV was the best threshold to depict complete pathological response obtaining a $85.4 \%$ of sensitivity and a $65.2 \%$ of specificity while they [35] did not identify the optimal cut-off for the late response after PCRT. Leccisotti et al. [35] concluded that the PET/CT can predict early pCRT response depicting non-complete responders and allowing modification of treatment; contrariwise, late response before surgery is not sufficiently accurate for guiding the surgical decision versus TME, conservative strategies or observation over time. Niccoli-Asabella et al. [36] reported similar findings. Kim et al. [37] demonstrated that post-CRT SUVmax had a sensitivity of $60.4 \%$, a specificity of $65.0 \%$, and an accuracy of $55.9 \%$. Palma et al. [38] reported that postCRT SUVmax had a sensitivity of $45.0 \%$, a specificity of $70.0 \%$, and an accuracy of $60.0 \%$. Similar results were observed on advanced esophageal cancer [39]. Overall these data show the poor accuracy of late metabolic response to predict pathological responses, while they support the usefulness of performing PET/CT early during preoperative CRT in LARC.

Using $\triangle$ SIS analysis, we obtained better results than $\triangle \mathrm{SUV}_{\max }$, both in terms of sensitivity $(92.7 \%)$, negative predictive value $(92,7 \%)$ and accuracy $(89.3 \%)$, considering the optimal threshold of $6.0 \%$. These results are comparable with the findings reported in our previous paper [13] where $\Delta$ SIS percentage variation obtained a sensitivity of $93.5 \%$ and a specificity of $82.1 \%$. $\Delta$ SIS showed a statistically significant difference in median values for responder and non-responder patients based on TRG and pathological $\mathrm{T}$ stage. In addition, a good linear correlation between $\triangle$ SIS median values and TRG score (Spearman's rank correlation coefficient $=-0.7$ ), was also observed.

Diagnostic performance of $\triangle \mathrm{SIS}$ to assess preoperative CRT response was statistically significant in comparison of $\triangle \mathrm{SUV}_{\max }$ performance resulting an increase of sensitivity of $25.4 \%$ and an increase of negative predictive value of $34.5 \%$ (McNemar test $p$ value $<0.05)$. Moreover, an increase of $\Delta$ SIS diagnostic performance respect to $\triangle \mathrm{SUVmax}$ was also observed in the differentiation of pathological complete response by incomplete response ( $\triangle$ SIS cut-off of $30 \%$ ): $13.3 \%$ of sensitivity increase, $37.8 \%$ of specificity increase, $23.1 \%$ of PPV increase and $23.9 \%$ of NPV increase. However, ${ }^{18} \mathrm{~F}$-FDG PET/CT evaluation remains a more widely applicable approach to predict neo-adjuvant therapy response in LARC, whereas SIS is for the time being a promising DCE-MRI angiogenic biomarker with great potential for assessing preoperative treatment response and directing surgery for more or less conservative treatment.

The heterogeneity in the neoadjuvant treatment scheme with the majority of study population receiving an experimental schedule of "antiangiogenic" agent plus oxaliplatin in comparison of standard CRT scheme was previously investigated in the our study [13]. The analysis in [13] showed that the treatment schedule did not influence the proportions in responder and non-responder patients.

Some potential limitations deserve a special consideration: two radiologists assessed the MR images in agreement and in a single session per patient so that
A

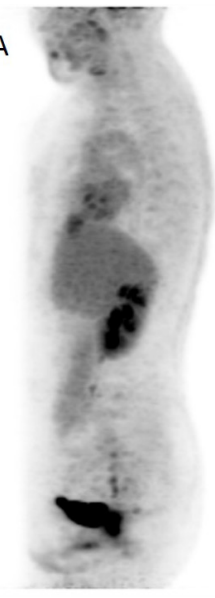

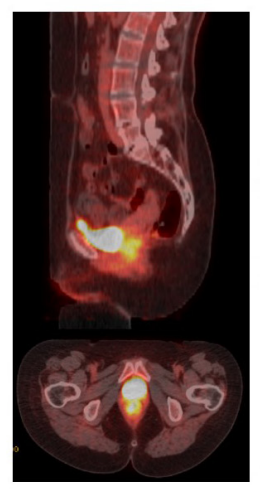

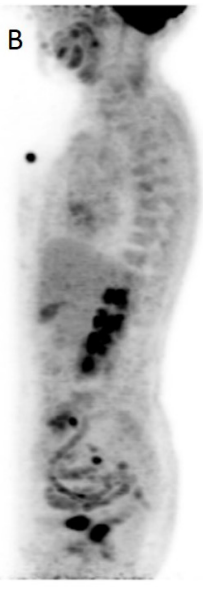

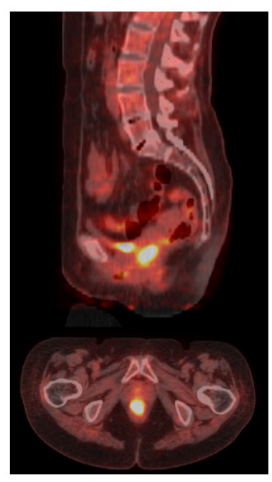

Figure 5: ${ }^{18} \mathrm{~F}-\mathrm{FDG}$ PET/CT images before A and after $\mathbf{B}$ treatment demonstrating a minimal reduction of glucose metabolism with $\triangle \mathrm{SUV}_{\max }$ of $6.07 \%$ classifying the patient as non responder. 
Table 4: Pulse Sequence Parameters on MR studies

\begin{tabular}{llcccccc}
\hline Sequence & Orientation & $\begin{array}{c}\text { TR/TE/FA } \\
(\mathbf{m s} / \mathbf{m s} / \mathbf{d e g})\end{array}$ & AT (min) & $\begin{array}{c}\text { FOV } \\
(\mathbf{m m x m m})\end{array}$ & Matrix & $\begin{array}{c}\text { ST/Gap } \\
(\mathbf{m m} / \mathbf{m m})\end{array}$ & TF \\
\hline T1w 2D TSE & Coronal & $499 / 13 / 150$ & 2.36 & $450 \times 450$ & $256 \times 230$ & $3 / 0$ & 3 \\
T2w 2D TSE & Sagittal & $4820 / 98 / 150$ & 4.17 & $260 \times 236$ & $256 \times 139$ & $3 / 0$ & 13 \\
T2w 2D TSE & Coronal & $4820 / 98 / 150$ & 4.17 & $260 \times 236$ & $256 \times 139$ & $3 / 0$ & 13 \\
T2w 2D TSE & Axial & $3970 / 98 / 150$ & 3.48 & $270 \times 236$ & $256 \times 157$ & $3 / 0$ & 13 \\
T1w FLASH 3D & Axial & $9.8 / 4.76 / 25$ & 0.58 & $330 \times 247$ & $256 \times 192$ & $3 / 0$ & $/$ \\
T1w FLASH 3D & Axial & $9.8 / 4.76 / 25$ & $0.58 \times 10$ & $330 \times 247$ & $256 \times 192$ & $3 / 0$ & $/$ \\
T1w 2D TSE & Sagittal & $538 / 13 / 150$ & 2.35 & $250 \times 250$ & $256 \times 230$ & $3 / 0$ & 5 \\
T1w 2D TSE & Coronal & $538 / 13 / 150$ & 2.52 & $250 \times 250$ & $256 \times 230$ & $3 / 0$ & 5 \\
T1w 2D TSE & Axial & $450 / 12 / 150$ & 2.31 & $270 \times 236$ & $256 \times 202$ & $3 / 0$ & 5 \\
\hline
\end{tabular}

Abbreviations: $\mathrm{TR}=$ Repetition Time, $\mathrm{TE}=$ Echo Time, $\mathrm{FOV}=$ Field of View, FA $=$ Flip Angle, $\mathrm{ST}=$ Slice Thickness, $\mathrm{TF}=$ Turbo Factor, $\mathrm{AT}=$ Acquisition Time

the intra-observer variability analysis was not performed. Butylscopolamine, dicyclomine, glucagon or similar drugs were not administered; however we performed volumetric analysis that minimize errors due to caused voxel misalignments.

Future improvement of this application could be the 1) development of an easy to use and user friendly SIS evaluation software, 2) comparison of SIS analysis with diffusion and perfusion coefficients obtained by Diffusion Weighted Imaging data analysis, 3) combination of multiple functional biomarkers (SIS, SUV, Diffusion Coefficients) to early predict neoadjuvant therapy response in LARC.

In conclusion, our study proposes an imaging angiogenetic biomarker, the Standardized Index of Shape, as an objective measurable index, easily transferable to clinical routine through a user-friendly software application, able to assess pCRT tumor response with a reproducible semi-quantitative measure of tumor blood perfusion. SIS percentage change could play an important role in LARC management helping to identify significant pathological response in order to adopt conservative strategies and to detect complete pathological response in order to guide versus a "wait and see" policy, reducing substantial morbidity and functional complications of TME.

\section{MATERIALS AND METHODS}

\section{Patient selection}

75 consecutive patients - with a median age of 62 years (range 44-77 years) were enrolled in this prospective study, from March 2007 to June 2014. All patients had a biopsy-proven rectal adenocarcinoma. Endorectal ultrasonography, pelvis MRI and whole body contrast enhanced $\mathrm{CT}$ scans were used for staging. Inclusion criteria were: patients with clinical T3-4 or with nodal involvement. Exclusion criteria were: inability to give informed consent, previous rectal surgery and contraindications for undergoing MRI or administering MR contrast media. Fifty-four (72\%) patients had been enrolled in a phase II prospective trial previously described [9]. The study was approved by the Independent Ethical Committee of our institution. All patients gave written informed consent to participate to the study.

\section{Neoadjuvant therapy and surgical approach}

External radiation therapy was performed using a 3 -field technique (one posterior-anterior and two lateral fields). Standard fractions of $1.8 \mathrm{~Gy} /$ day to the reference point were given, 5 times a week up to a total dose of 45 Gy. Details of treatment planning have been previously reported [9]. Fifty-four patients received an experimental treatment with biweekly bevacizumab at $5 \mathrm{mg} / \mathrm{kg}$ plus three biweekly cycles of oxaliplatin at $100 \mathrm{mg} / \mathrm{m}^{2}$ and raltitrexed at $2.5 \mathrm{mg} / \mathrm{m}^{2}$ on day 1 , and levo-folinic acid at $250 \mathrm{mg} / \mathrm{m}^{2}$, and 5 -Fluorouracil at $800 \mathrm{mg} / \mathrm{m}^{2}$ on day 2 [8]. 21 remaining subjects received standard treatment with capecitabine at a dose of $825 \mathrm{mg} / \mathrm{m}^{2}$ twice daily, 5 days a week, for 5 weeks.

Patients underwent TME $8 \quad( \pm 1)$ weeks after completing CRT. An anterior or abdominoperineal resection was performed on the basis of the results of restaging.

\section{FDG-PET data acquisition and analysis}

PET studies were acquired $60 \mathrm{~min}$ after the administration of 300-385 MBq of FDG either with a General Electric Discovery DST 600 PET/CT scanner 
[10]. All calibrations on the scanners to obtain accurate SUV readings were regularly performed. Patients fasted for at least $6 \mathrm{~h}$, and blood glucose level was $<150 \mathrm{mg} / \mathrm{dl}$. Each patient underwent the baseline and the pre-operative study on the same scanner.

${ }^{18} \mathrm{~F}-\mathrm{FDG}$ PET/CT image assessment was performed in a single reading session for each patient by consensus of two expert investigator with at least 15 years of experience. The readers were blinded to the clinicopathologic outcome and MRI findings. Irregular volumes of interest (VOIs) were semi-automatically drawn on orthogonal planes using a dedicated workstation and software using an arbitrary threshold, as reported previously [10]. For each patient both studies were analyzed at the same time in order to minimize discrepancies in VOI positioning. For each study maximum SUV ( $\mathrm{SUV}_{\max }$ ) values of the rectal lesion were recorded. FDG PET analysis results was performed by comparing measurements obtained in the rectal lesion at baseline $\left(\mathrm{SUV}_{1}\right)$ and after treatment $\left(\mathrm{SUV}_{2}\right)$. This change (known also as response index) was expressed as the percentage of SUV reduction $(\triangle \mathrm{SUV}=$ $\left.\left(\mathrm{SUV}_{1}-\mathrm{SUV}_{2}\right) / \mathrm{SUV}_{1} \times 100\right)[9]$

\section{MRI data acquisitions}

All patients underwent DCE-MRI before and after CRT. Imaging was performed with a $1.5 \mathrm{~T}$ scanner (Magnetom Symphony, Siemens Medical System, Erlangen, Germany) equipped with a phased-array body coil. Patients were placed in a supine, head-first position. Mild rectal lumen distension was achieved with 60-90 mL of undiluted ferumoxil (Lumirem, Guerbet, Roissy CdG Cedex, France) suspension introduced per rectum in order to obtain mild distension of rectal lumen [21] and improve the evaluation of rectal wall involvement, particularly in the post contrast MR scan. Pre-contrast coronal T1w 2D turbo spin-echo images and sagittal, coronal and axial T2w 2D turbo spin-echo images of the pelvis were obtained. Subsequently, axial, dynamic, contrast-enhanced T1w, FLASH 3D gradient-echo images were acquired for semiquantitative MRI analysis. We obtained one sequence before and ten sequences, without any delay, after the IV injection of $0.2 \mathrm{~mL} / \mathrm{kg}$ of a positive, gadolinium-based paramagnetic contrast medium (Gd-DOTA, Dotarem, Guerbet, Roissy CdG Cedex, France). The contrast medium was administered using a Spectris Solaris ${ }^{\circledR}$ EP MR (MEDRAD Inc., Indianola, PA) injector, with a flow rate of $2 \mathrm{~mL} / \mathrm{s}$, followed by a $10-\mathrm{mL}$ saline flush at the same rate. Sagittal, axial and coronal post-contrast T1w 2D turbo spin-echo images, with and without fat saturation were obtained. The axial images were acquired without any angulation. Axial T1-w pre- and post-contrast sequences were acquired at the same position as the T2-W sequence. MRI total acquisition time was around 30 minutes. Sequence parameters details were reported in Table 4.
Spin-echo diffusion-weighted echo-planar imaging at different $b$ values was performed for a limited subgroups of patients for this reason is not effected its analysis in this manuscript but could be object of a future study.

\section{MRI image data analysis}

Image assessment was performed in a single reading session for each patient by consensus of two gastrointestinal radiologists with 13 years and 5 years of experience in reading pelvic MR images. MRI readers were blinded to the clinicopathologic outcome and PET/ CT findings.

Regions of interest (ROIs) to cover the entire tumor volume were manually drawn slice by slice on pre-contrast T1-weighted images using the T2-weighted images as a guide [22]. Attention was placed to cover the entire lesion with the exclusion of peripheral fat, artefacts and blood vessels. Median values were recorded for all acquired tumor slices for each study.

For SIS analysis, for each TIC, the maximum signal difference (MSD) and wash out slope (WOS) were calculated, considering median value percentage change $\left(\Delta \mathrm{MSD}=\left(\mathrm{MSD}_{1}-\mathrm{MSD}_{2}\right) / \mathrm{MSD}_{1} \times 100\right.$ and $\Delta \mathrm{WOS}=\left(\mathrm{WOS}_{1}\right.$ $-\mathrm{WOS}_{2}$ )/WOS $\times 100$ ), their linear combination (equation 1) was evaluated as described in our previously paper [13]:

$$
\Delta S I S=0.7780 * \Delta \mathrm{MSD}+0.6157 * \Delta \mathrm{WOS}
$$

Semi-quantitative analyses were carried out using Matlab R2007a software (The Math-Works Inc., Natick, MA).

\section{Evaluation of pathologic response}

Details of how pathologic response assessment was performed have been described [8]. Briefly, surgical specimens containing the tumour were evaluated and scored according to tumour regression grade (TRG), as proposed by Mandard et al. [24] by two experienced pathologists who were not aware of MRI and FDG PET findings. Patients with a TRG 1 or 2 score were considered responders, whereas the remaining patients (TRG 3, 4, or 5) were classified as non responders.

\section{Statistical analysis}

All quantitative data values were expressed as median \pm standard deviation (SD) and compared with Mann-Whitney test. Chi-square test was performed to evaluate differences between pathologic responders (TRG 1-2) and non-responders (TRG 3-4) regarding baseline patient and tumour characteristics. Receiver operating characteristic (ROC) curves were calculated using $\triangle$ SIS and $\triangle \mathrm{SUV}_{\max }$ and optimal thresholds were obtained maximizing the Youden index. Sensitivity, specificity, positive and negative predictive value (PPV and NPV), 
for $\Delta \mathrm{SIS}$ and $\Delta \mathrm{SUV}$, to differentiate responders by non responders patient and pathological complete response (TRG1) by incomplete response (TRG2-4) were performed. Matched sample tables and the McNemar Chi-square tests were used to compare the performance. Spearman's rank correlation coefficient was used to evaluate correlation between $\Delta \mathrm{SIS}$ and $\Delta \mathrm{SUV}_{\text {max }}$ with TRG and pathological $\mathrm{T}$ stage (pT).

$\mathrm{P}$ value $<0.05$ was considered significant for all tests. All analyses were performed using Statistics Toolbox of Matlab R2007a (The Math-Works Inc., Natick, MA).

\section{CONFLICTS OF INTEREST}

The authors have no conflict of interest to be disclosed.

\section{REFERENCES}

1. Siegel RL, Miller KD, Jemal A. Cancer statistics, 2015. CA Cancer J Clin. 2015 Jan-Feb; 65:5-29.

2. Avallone A, Aloj L, Aprile G, Rosati G, Budillon A. A perspective on the current treatment strategies for locally advanced rectal cancer. Int J Biochem Cell Biol. 2015 Aug; 65:192-6.

3. Avallone A, Delrio P, Pecori B, Tatangelo F, Petrillo A, Scott N, Marone P, Aloi L, Sandomenico C, Lastoria S, Iaffaioli VR, Scala D, Iodice G, et al. Oxaliplatin plus dual inhibition of thymidilate synthase during preoperative pelvic radiotherapy for locally advanced rectal carcinoma: long-term outcome. Int J Radiat Oncol Biol Phys. 2011 Mar 1; 79:670-6

4. Petrillo M, Fusco R, Catalano O, Sansone M, Avallone A, Delrio P, Pecori B, Tatangelo F, Petrillo A. MRI for Assessing Response to Neoadjuvant Therapy in Locally Advanced Rectal Cancer Using DCE-MR and DW-MR Data Sets: A Preliminary Report. Biomed Res Int. 2015; 2015:514740.

5. Beets-Tan RG, Beets GL. Rectal cancer: review with emphasis on MR imaging. Radiology 2004; 232:335-346.

6. Cascini GL, Avallone A, Delrio P, Guida C, Tatangelo F, Marone P, Aloj L, De Martinis F, Comella P, Parisi V, Lastoria S. 18F-FDG PET is an early predictor of pathologic tumor response to preoperative radiochemotherapy in locally advanced rectal cancer. J Nucl Med 2006; 47:1241-8.

7. Huh JW, Kwon SY, Lee JH, Kim HR. Comparison of restaging accuracy of repeat FDG-PET/CT with pelvic MRI after preoperative chemoradiation in patients with rectal cancer. J Cancer Res Clin Oncol. 2015 Feb; 141:353-9.

8. Aiba $T$, Uehara $K$, Nihashi $T$, Tsuzuki T, Yatsuya $H$, Yoshioka Y, Kato K, Nagino M. MRI and FDG-PET for assessment of response to neoadjuvant chemotherapy in locally advanced rectal cancer. Ann Surg Oncol. 2014 Jun; 21:1801-8.
9. Avallone A, Pecori B, Bianco F, Aloj L, Tatangelo F, Romano C, Granata V, Marone P, Leone A, Botti G, Petrillo A, Caracò C, Iaffaioli VR, et al. Critical role of bevacizumab scheduling in combination with pre-surgical chemo-radiotherapy in MRI-defined high-risk locally advanced rectal cancer: Results of the BRANCH trial. Oncotarget. 2015; 6:30394-407. doi: 10.18632/ oncotarget.4724.

10. Avallone A, Aloj L, Caracò C, Delrio P, Pecori B, Tatangelo F, Scott N, Casaretti R, Di Gennaro F, Montano M, Silvestro L, Budillon A, Lastoria S. Early FDG PET response assessment of preoperative radiochemotherapy in locally advanced rectal cancer: correlation with long-term outcome. Eur J Nucl Med Mol Imaging. 2012 Dec; 39:1848-57.

11. Beets-Tan RG, Beets GL. MRI for assessing and predicting response to neoadjuvant treatment in rectal cancer. Nat Rev Gastroenterol Hepatol. 2014 Aug; 11:480-8.

12. Petrillo A, Fusco R, Petrillo M, Granata V, Filice S, Delrio P, Tatangelo F, Pecori B, Botti G, Muto P, Romano G, Avallone A. Dynamic Contrast Enhanced-MRI in locally advanced rectal cancer: Value of Time Intensity Curve Visual Inspection to assess neo-adjuvant therapy response. The Journal of Physiology and Health. 2014; 255-267.

13. Petrillo A, Fusco R, Petrillo M, Granata V, Sansone M, Avallone A, Delrio P, Pecori B, Tatangelo F, Ciliberto G. Standardized Index of Shape (SIS): a quantitative DCE-MRI parameter to discriminate responders by nonresponders after neoadjuvant therapy in LARC. Eur Radiol. 2015 Jul; 25:1935-45.

14. Daniel BL, Yen YF, Glover GH, Ikeda DM, Birdwell RL, Sawyer-Glover AM, Black JW, Plevritis SK, Jeffrey SS, Herfkens RJ. Breast disease: dynamic spiral MR imaging. Radiology 1998; 209:499-509.

15. Gollub MJ, Gultekin DH, Akin O, Do RK, Fuqua JL, Gonen M, Kuk D, Weiser M, Saltz L, Schrag D, Goodman K, Paty $\mathrm{P}$, Guillem J, et al. Dynamic contrast enhanced-MRI for the detection of pathological complete response to neoadjuvant chemotherapy for locally advanced rectal cancer. Eur Radiol. 2012 Apr; 22:821-31.

16. Hayes C, Padhani AR, Leach MO. Assessing changes in tumour vascular function using dynamic contrast-enhanced magnetic resonance imaging. NMR Biomed 2002; 15:154-63.

17. Zhang XM, Yu D, Zhang HL, Dai Y, Bi D, Liu Z, Prince MR, Li C. 3D dynamic contrast-enhanced MRI of rectal carcinoma at 3T: correlation with microvascular density and vascular endothelial growth factor markers of tumor angiogenesis. J Magn Reson Imaging 2008; 27:1309-1316.

18. Fusco R, Sansone M, Maffei S, Petrillo. A. Dynamic Contrast-Enhanced MRI in Breast Cancer: A Comparison between Distributed and Compartmental Tracer Kinetic Models. Journal of Biomedical Graphics and Computing, Journal of Biomedical Graphics and Computing 2012; 2:23.

19. Fusco R, Petrillo A, Petrillo M, Sansone M. Use of Tracer Kinetic Models for Selection of Semi-Quantitative Features 
for DCE-MRI Data Classification. Applied Magnetic Resonance Sep 2013; 44:1311-1324.

20. Guo JY, Reddick WE. DCE-MRI pixel-by-pixel quantitative curve pattern analysis and its application to osteosarcoma. J Magn Reson Imaging 2009; 30:177-184.

21. Petrillo A, Catalano O, Delrio P, Avallone A, Guida C, Filice S, Siani A. Post-treatment fistulas in patients with rectal cancer: MRI with rectal superparamagnetic contrast agent. Abdom Imaging. 2007 May-Jun; 32:328-31.

22. Dresen RC, Beets GL, Rutten HJ, Engelen SM, Lahaye MJ, Vliegen RF, de Bruïne AP, Kessels AG, Lammering G, Beets-Tan RG. Locally advanced rectal cancer: MR imaging for restaging after neoadjuvant radiation therapy with concomitant chemotherapy. Part I. Are we able to predict tumor confined to the rectal wall? Radiology. 2009 Jul; 252:71-80.

23. Andreola S, Leo E, Belli F, Bonfanti G, Sirizzotti G, Greco P, Valvo F, Tomasic G, Gallino GF. Adenocarcinoma of the lower third of the rectum surgically treated with a $<10-\mathrm{MM}$ distal clearance: preliminary results in $35 \mathrm{~N} 0$ patients. Ann Surg Oncol. 2001 Aug; 8:611-5.

24. Mandard AM, Dalibard F, Mandard JC, Marnay J, HenryAmar M, Petiot JF, Roussel A, Jacob JH, Segol P, Samama $\mathrm{G}$, et al. Pathologic assessment of tumor regression after preoperative chemoradiotherapy of esophageal carcinoma. Clinicopathologic correlations. Cancer. 1994 Jun 1; 73:2680-6.

25. Gunderson LL, Callister M, Marschke R, Young-Fadok T, Heppell J, Efron J. Stratification of Rectal Cancer Stage for Selection of Postoperative Chemoradiotherapy: Current Status. Gastrointestinal Cancer Research: GCR. 2008; 2:25-33.

26. Vecchio FM, Valentini V, Minsky BD, Padula GD, Venkatraman ES, Balducci $M$, Miccichè F, Ricci R, Morganti AG, Gambacorta MA, Maurizi F, Coco C. The relationship of pathologic tumor regression grade (TRG) and outcomes after preoperative therapy in rectal cancer. Int J Radiat Oncol Biol Phys 2005; 62:752-60.

27. Borschitz T, Wachtlin D, Möhler M, Schmidberger H, Junginger T. Neoadjuvant chemoradiation and local excision for T2-3 rectal cancer. Ann Surg Oncol 2008; 15:712-20.

28. Habr-Gama A, Perez RO, Nadalin W, Sabbaga J, Ribeiro U Jr, Silva e Sousa AH Jr, Campos FG, Kiss DR, GamaRodrigues J. Operative versus nonoperative treatment for stage 0 distal rectal cancer following chemoradiation therapy: long-term results. Ann Surg. 2004 Oct; 240:711-8.

29. Lezoche G, Baldarelli M, Guerrieri M, Paganini AM, De Sanctis A, Bartolacci S, Lezoche E. A prospective randomized study with a 5-year minimum follow-up evaluation of transanal endoscopic microsurgery versus laparoscopic total mesorectal excision after neoadjuvant therapy. Surg Endosc. 2008 Feb; 22:352-8.

30. Martens MH, Subhani S, Heijnen LA, Lambregts DM, Buijsen J, Maas M, Riedl RG, Jeukens CR, Beets GL, Kluza
E, Beets-Tan RG. Can perfusion MRI predict response to preoperative treatment in rectal cancer? Radiother Oncol. 2015 Feb; 114:218-23.

31. Tong T, Sun Y, Gollub MJ, Peng W, Cai S, Zhang $\mathrm{Z}, \mathrm{Gu}$ Y. Dynamic contrast-enhanced MRI: Use in predicting pathological complete response to neoadjuvant chemoradiation in locally advanced rectal cancer. J Magn Reson Imaging. 2015 Sep; 42:673-80.

32. Patterson DM, Padhani AR, Collins DJ. Technology insight: water diffusion MRI - a potential new biomarker of response to cancer therapy. Nat Clin Pract Oncol 2008; 5:220-33.

33. Ippolito D, Fior D, Trattenero C, Ponti ED, Drago S, Guerra L, Franzesi CT, Sironi S. Combined value of apparent diffusion coefficient-standardized uptake value max in evaluation of post-treated locally advanced rectal cancer. World J Radiol. 2015 Dec 28; 7:509-20.

34. Joye I, Deroose CM, Vandecaveye V, Haustermans K. The role of diffusion-weighted MRI and (18)F-FDG PET/CT in the prediction of pathologic complete response after radiochemotherapy for rectal cancer: a systematic review. Radiother Oncol. 2014 Nov; 113:158-65.

35. Leccisotti L, Gambacorta MA, de Waure C, Stefanelli A, Barbaro B, Vecchio FM, Coco C, Persiani R, Crucitti A, Tortorelli AP, Giordano A, Valentini V. The predictive value of $18 \mathrm{~F}-\mathrm{FDG} \mathrm{PET} / \mathrm{CT}$ for assessing pathological response and survival in locally advanced rectal cancer after neoadjuvant radiochemotherapy. Eur J Nucl Med Mol Imaging. 2015 Apr; 42:657-66.

36. Niccoli-Asabella A, Altini C, De Luca R, Fanelli M, Rubini D, Caliandro C, Montemurro S, Rubini G. Prospective analysis of $18 \mathrm{~F}-\mathrm{FDG}$ PET/CT predictive value in patients with low rectal cancer treated with neoadjuvant chemoradiotherapy and conservative surgery. Biomed Res Int. 2014; 2014:952843.

37. Kim JW, Kim HC, Park JW, Park SC, Sohn DK, Choi HS, Kim DY, Chang HJ, Baek JY, Kim SY, Kim SK, Oh JH. Predictive value of (18)FDG PET-CT for tumour response in patients with locally advanced rectal cancer treated by preoperative chemoradiotherapy. Int J Colorectal Dis. 2013 Sep; 28:1217-24.

38. Palma P, Conde-Muíño R, Rodríguez-Fernández A, SeguraJiménez I, Sánchez-Sánchez R, Martín-Cano J, Gómez-Río M, Ferrón JA, Llamas-Elvira JM. The value of metabolic imaging to predict tumour response after chemoradiation in locally advanced rectal cancer. Radiat Oncol. 2010 Dec $15 ; 5: 119$.

39. Wieder HA, Brucher BL, Zimmermann F, Becker K, Lordick F, Beer A, Schwaiger M, Fink U, Siewert JR, Stein HJ, Weber WA. Time course of tumor metabolic activity during chemoradiotherapy of esophageal squamous cell carcinoma and response to treatment. J Clin Oncol. 2004; 22:900-908. 